\title{
ON UNIFORM BOUNDS OF PRIMENESS IN MATRIX RINGS
}

\author{
KONSTANTIN I. BEIDAR and ROBERT WISBAUER
}

(Received 20 October 2001; revised 19 November 2002)

\author{
Communicated by J. Du
}

\begin{abstract}
A subset $\mathscr{S}$ of an associative ring $R$ is a uniform insulator for $R$ provided $a \mathscr{S} b \neq 0$ for any nonzero $a, b \in R$. The ring $R$ is called uniformly strongly prime of bound $m$ if $R$ has uniform insulators and the smallest of those has cardinality $m$. Here we compute these bounds for matrix rings over fields and obtain refinements of some results of van den Berg in this context.

More precisely, for a field $F$ and a positive integer $k$, let $m$ be the bound of the matrix $\operatorname{ring} M_{k}(F)$, and let $n$ be $\operatorname{dim}_{F}(\mathscr{V})$, where $\mathscr{V}$ is a subspace of $M_{k}(F)$ of maximal dimension with respect to not containing rank one matrices. We show that $m+n=k^{2}$. This implies, for example, that $n=k^{2}-k$ if and only if there exists a (nonassociative) division algebra over $F$ of dimension $k$.
\end{abstract}

2000 Mathematics subject classification: primary $16550,16 \mathrm{~N} 60$.

\section{Introduction}

Following Handelman and Lawrence [1, page 211], we call a subset $\mathscr{S}$ of an associative ring $\mathscr{R}$ a uniform insulator for $\mathscr{R}$ if $a \mathscr{S} b \neq 0$ for all $a, b \in \mathscr{R}$ with $a \neq 0 \neq b$. The ring $\mathscr{R}$ is said to be uniformly strongly prime if it contains a finite uniform insulator. For such a ring we set $m(\mathscr{R})=\min \{|\mathscr{S}| \mid \mathscr{S}$ is a uniform insulator of $\mathscr{R}\}$, and we say $\mathscr{R}$ is uniformly strongly prime of bound $n$ provided $m(\mathscr{R})=n$.

In what follows $F$ is a field and $M_{k}(F)$ stands for the algebra of $k \times k$ matrices over $F$, where $k$ is a positive integer. Note that $M_{k}(F)$ is always uniformly strongly prime in view of [2, Theorem 3] (or [3, Theorem 1]). For $\mathscr{R}=M_{k}(F)$ we put $m_{k}(F):=m(\mathscr{R})$.

The systematic study of $m(\mathscr{R})$ was initiated by van den Berg in $[2,3]$ and we recall the following of his results ([3, Theorems $4,7,11])$.

(C) 2004 Australian Mathematical Society $1446-7887 / 04 \$ A 2.00+0.00$ 
THEOREM 1.1.

(i) Let $\mathscr{D}$ be a division ring and $\mathscr{R}=M_{k}(\mathscr{D})$. Then $k \leq m(\mathscr{R}) \leq 2 k-1$.

(ii) If $F$ is an algebraically closed field, then $m_{k}(F)=2 k-1$.

(iii) Let $F$ be a field and assume there exists a nonassociative division $F$-algebra of dimension $k$, then $m_{k}(F)=k$.

In [3, Remark 2], van den Berg asks if the converse of assertion (iii) holds. In the present paper we obtain a positive answer to this question (see Corollary 1.4 (iii)). We sharpen the above results by studying connections of the uniform bound of $M_{k}(F)$ with (maximal) dimension of certain subspaces of $M_{k}(F)$ and $M_{k^{2}}(F)$. We also pose some open questions.

Before stating our results we fix some notation. Given positive integers $k, l$ we denote by $M_{k, l}(F)$ the $k \times l$-matrices over the field $F$.

For $A=\left(a_{i j}\right)_{1 \leq i \leq k, 1 \leq j \leq l} \in M_{k, l}(F)$ and $B \in M_{l, k}(F)$, we define

$$
A \bullet B=\left(\begin{array}{cccc}
a_{11} B & a_{12} B & \cdots & a_{1 l} B \\
a_{21} B & a_{22} B & \cdots & a_{2 l} B \\
\cdots & \ldots & \cdots & \cdots \\
a_{k 1} B & a_{k 2} B & \cdots & a_{k l} B
\end{array}\right) \in M_{k l}(F) .
$$

If $l=1$, then $A \bullet B=A B$, and it is known that a matrix $C \in M_{k}(F)$ has rank one if and only if there exist nonzero matrices $A \in M_{k, 1}(F)$ and $B \in M_{1, k}(F)$ such that $C=A B=A \bullet B$.

If $l=k$, it is well known that $\phi: M_{k}(F) \otimes_{F} M_{k}(F) \rightarrow M_{k^{2}}(F)$, the linear extension of the map $A \otimes B \mapsto A \bullet B$, is an algebra isomorphism.

With this in mind we introduce the following entities which will be helpful for our purposes:

$$
\begin{aligned}
& n_{k}(F)=\max \left\{\begin{array}{l|l}
\operatorname{dim}_{F}(\mathscr{V}) & \begin{array}{l}
\mathscr{V} \text { is a subspace of } M_{k}(F) \text { and } \\
\mathscr{V} \cap\left\{M_{k, 1}(F) \bullet M_{1, k}(F)\right\}=0
\end{array}
\end{array}\right\}, \\
& l_{k}(F)=\max \left\{\begin{array}{l|l}
\operatorname{dim}_{F}(\mathscr{K}) & \begin{array}{l}
\mathscr{K} \subseteq M_{k^{2}}(F) \text { is a left ideal and } \\
\mathscr{K} \cap\left\{M_{k}(F) \bullet M_{k}(F)\right\}=0
\end{array}
\end{array}\right\} .
\end{aligned}
$$

We are now in a position to state the main results of the present paper.

THEOREM 1.2. Given a field $F$ and positive integer $k$, we have:

(i) $m_{k}(F)=2 k-1$, for all $k$, if and only if $F$ is algebraically closed.

(ii) $m_{k}(F)=k$ if and only if there exists a nonassociative division $F$-algebra of dimension $k$.

The above result sharpens (ii) and (iii) in Theorem 1.1. We note that the theorem is essentially a corollary to van den Berg's results. The next observations provide relationships between the dimensions under consideration. 
THEOREM 1.3. Given a field $F$ and positive integer $k$, we have $m_{k}(F)+n_{k}(F)=k^{2}$ and $l_{k}(F)=k^{2} \cdot n_{k}(F)$.

We list some immediate implications.

COROLLARY 1.4. Let $\mathscr{V}$ be a $k$ dimensional vector space over a field $F$ and let $\bar{F}$ be the algebraic closure of $F$. Then:

(i) $k^{2}-2 k+1 \leq n_{k}(F) \leq k^{2}-k$.

(ii) $n_{k}(F)=k^{2}-2 k+1$, for all $k$, if and only if $F$ is algebraically closed.

(iii) $n_{k}(F)=k^{2}-k$ if and only if there exists a nonassociative division $F$-algebra of dimension $k$.

(iv) A subspace $\mathscr{W} \subset M_{k}(F)$ contains a rank one matrix, provided $\operatorname{dim}_{F}(\mathscr{W})>$ $k^{2}-k$, or $F=\bar{F}$ and $\operatorname{dim}_{F}(\mathscr{W})>k^{2}-2 k+1$.

(v) A subspace $\mathscr{W} \subset \mathscr{V} \otimes_{F} \mathscr{V}$ contains a non-zero element of the form $A \otimes B$ for some $A, B \in \mathscr{V}$, provided $\operatorname{dim}(\mathscr{W})>k^{2}-k$, or $F=\bar{F}$ and $\operatorname{dim}(\mathscr{W})>k^{2}-2 k+1$.

PROOF. (i) follows at once from Theorem 1.1 and Theorem 1.3. (ii) and (iii) are immediate consequences of Theorem 1.2 (ii) together with Theorem 1.3. (iv) follows from (i) and (ii). Clearly $\mathscr{V} \cong M_{k 1}(F)$ and $\mathscr{V} \cong M_{1 k}(F)$ as vector spaces. Next, the linear extension of the map $A \otimes B \mapsto A B, A \in M_{k 1}(F), B \in M_{1 k}(F)$, is an isomorphism of vector spaces $M_{k 1}(F) \otimes_{F} M_{1 k}(F) \rightarrow M_{k}(F)$. Therefore there exists an isomorphism $\mathscr{V} \otimes_{F} \mathscr{V} \rightarrow M_{k}(F)$ of vector spaces sending vectors of the form $v \otimes u$ to matrices of rank 1 . The result now follows from (iv).

\section{Proof of the main theorems}

Given a division ring $\mathscr{D}$ and a positive integer $k$, we denote by $G L(k ; \mathscr{D})$ the group of invertible $k \times k$ matrices over $\mathscr{D}$. We need the following result.

COROLlaRY 2.1 ([3, Corollary 5]). The following assertions are equivalent for a division ring $\mathscr{D}$ and a positive integer $k$ :

(i) $M_{k}(\mathscr{D})$ is uniformly strongly prime of bound $k$.

(ii) $G L(k ; \mathscr{D}) \cup\{0\}$ contains a $k$-dimensional $\mathscr{D}$-subspace of $M_{k}(\mathscr{D})$.

Recall that a nonassociative $F$-algebra $\mathscr{D}$ is said to be a division algebra provided that for any $a, b \in \mathscr{D}$ with $a \neq 0$ both equations $a x=b$ and $y a=b$ have unique solutions in $\mathscr{D}$. We are now in a position to prove Theorem 1.2.

PROOF OF THEOREM 1.2. (i) If $F$ is algebraically closed, then $m_{k}(F)=2 k-1$ by Theorem 1.1. Conversely, if $F$ is not algebraically closed, then it has a finite extension $\mathscr{E}$ of dimension $k>1$. Therefore, $m_{k}(F)=k<2 k-1$ by Theorem 1.1 (iii). 
(ii) If there exists a nonassociative division $F$-algebra of dimension $k$, then $m_{k}(F)=k$ by Theorem 1.1 (iii). Conversely, assume that $m_{k}(F)=k$. Then Corollary 2.1 yields that $G L(k ; F) \cup\{0\}$ contains a $k$-dimensional $F$-subspace $\mathscr{V}$ of $M_{k}(F)$. Considering $M_{k}(F)$ as the endomorphism algebra of the vector space $\mathscr{V}$, we define a product $:: \mathscr{V} \times \mathscr{V} \rightarrow \mathscr{V}$ by the rule $A B=A(B)$ for all $A, B \in \mathscr{V}$. We claim that $(\mathscr{V}, \cdot)$ is a nonassociative division algebra over $F$ of dimension $k$. Indeed, let $A, B \in \mathscr{V}$ with $A \neq 0$. Consider the map $\phi: \mathscr{V} \rightarrow \mathscr{V}$ given by $\phi(X)=X A=X(A)$. Clearly $\phi$ is an endomorphism of the vector space $\mathscr{V}$. Since $\mathscr{V} \backslash\{0\} \subseteq G L(k ; F)$ and $A \neq 0, X(A) \neq 0$ for all $X \in \mathscr{V}$ with $X \neq 0$. That is $\operatorname{ker}(\phi)=0$ and so $\phi$ is an automorphism of $\mathscr{V}$. In particular, there exists a unique $Y \in \mathscr{V}$ such that $Y A=B$. Finally, since $A \in G L(k ; F)$, there exists a unique $X \in \mathscr{V}$ with $A X=A(X)=B$. Thus $(\mathscr{V}, \cdot)$ is a nonassociative division algebra and the proof is complete.

Let $\operatorname{tr}_{k}: M_{k}(F) \rightarrow F$ be the trace map. Given a subspace $\mathscr{W} \subseteq M_{k}(F)$, we set

$$
\mathscr{W}^{\perp}=\left\{A \in M_{k}(F) \mid \operatorname{tr}_{k}(A \mathscr{W})=0\right\} .
$$

Given $A \in M_{k, l}(F)$ and $B \in M_{l, k}(F)$, one can easily check that

$$
\operatorname{tr}_{\mathrm{k}}(A B)=\operatorname{tr}_{l}(B A)
$$

LEMMA 2.2. Let $\mathscr{W} \subseteq M_{k}(F)$ be a subspace containing no rank one matrices. Then any basis of $\mathscr{W}^{\perp}$ is a uniform insulator for $M_{k}(F)$. Conversely, let $\mathscr{S}$ be a uniform insulator for $M_{k}(F)$ and let $\mathscr{V}=\sum_{A \in \mathscr{S}} F A$. Then $\mathcal{Y}^{\downarrow}$ contains no rank one matrices.

PROOF. It is well known that the map $(A, B) \mapsto \operatorname{tr}_{k}(A B), A, B \in M_{k}(F)$, is a nondegenerate symmetric bilinear form. Therefore,

$$
\operatorname{dim}_{F}(\mathscr{U})+\operatorname{dim}_{F}\left(\mathscr{U}^{\perp}\right)=k^{2} \text { and }\left\{\mathscr{U}^{\perp}\right\}^{\perp}=\mathscr{U}
$$

for any subspace $\mathscr{U} \subseteq M_{k}(F)$.

Let $\mathscr{W}$ be as in the lemma and let $\mathscr{S}$ be a basis of $\mathscr{W}^{\perp}$. Given $0 \neq A \in M_{k, 1}(F)$ and $0 \neq B \in M_{1, k}(F), A B \in M_{k}(F)$ has rank one and so $A B \notin \mathscr{W}=\left\{\mathscr{W}^{\perp}\right\}^{\perp}$ forcing $0 \neq \operatorname{tr}_{k}(A B X)$ for some $X \in \mathscr{S}$. Making use of (1), we conclude that $B X A=\operatorname{tr}_{1}(B X A) \neq 0$. We see that $B \mathscr{S} A \neq 0$ for all $0 \neq A \in M_{k, 1}(F)$ and $0 \neq B \in M_{1, k}(F)$. Now let $P, Q \in M_{k}(F)$ be nonzero. Write

$$
P=\left(\begin{array}{c}
P_{1} \\
P_{2} \\
\vdots \\
P_{k}
\end{array}\right) \quad \text { and } \quad Q=\left(Q^{1}, Q^{2}, \ldots, Q^{k}\right)
$$


where $P_{i} \in M_{1, k}(F)$ and $Q^{j} \in M_{k, 1}(F)$. Then $P X Q=\left(P_{i} X Q^{j}\right)_{i, j=1}^{k}$ for all $X \in \mathscr{S}$ and so $P \mathscr{S} Q \neq 0$. Therefore $\mathscr{S}$ is a uniform insulator for $M_{k}(F)$.

Now let $\mathscr{S}$ and $\mathscr{V}$ be as in the lemma. Assume to the contrary that $\mathscr{V}^{\perp}$ contains a matrix $C$ of rank one. Write $C=A B$ where $A \in M_{k, 1}(F)$ and $B \in M_{1, k}(F)$. Clearly $A \neq 0$ and $B \neq 0$ (otherwise $C=0$ would be of rank 0 ). Since $A B=C \in \mathscr{V}^{\perp}$, $B X A=\operatorname{tr}_{1}(B X A)=\operatorname{tr}_{k}(A B X)=0$ for all $X \in \mathscr{S}$. Let $P, Q \in M_{k}(F)$ be matrices such that the first row of $P$ is equal to $B$ and all the other ones are equal to 0 , the first column of $Q$ is equal to $A$ and all the other ones are equal to 0 . Clearly $P \neq 0 \neq Q$ and $P \mathscr{S} Q=0$, a contradiction. The proof is thereby complete.

We denote by $A \mapsto{ }^{t} A, A \in M_{k}(F)$, the transpose map of $M_{k}(F)$. Define an action of $M_{k}(F) \otimes_{F} M_{k}(F)$ on $M_{k}(F)$ by the rule

$$
U X=\left(\sum_{i=1}^{n} A_{i} \otimes B_{i}\right) X=\sum_{i=1}^{n} A_{i} X^{t} B_{i}
$$

whenever $U=\sum_{i=1}^{n} A_{i} \otimes B_{i}$. It is well known that $M_{k}(F)$ is a simple faithful left module over the algebra $M_{k}(F) \otimes_{F} M_{k}(F)$ under this action and $M_{k}(F) \otimes_{F} M_{k}(F)$ is the algebra of all linear transformations of the vector space $M_{k}(F)$.

LEMMA 2.3. With the above notation we have:

(i) If $\mathscr{S}$ is a finite uniform insulator for $M_{k}(F)$ such that the set $\mathscr{S}$ is linearly independent over $F$, then $\mathscr{K}=\left\{U \in M_{k}(F) \otimes_{F} M_{k}(F) \mid U \mathscr{S}=0\right\}$ is a left ideal in $M_{k}(F) \otimes_{F} M_{k}(F)$ containing no nonzero elements of the form $A \otimes B, A, B \in M_{k}(F)$, and $\operatorname{dim}_{F}(\mathscr{K})=k^{2}\left(k^{2}-|\mathscr{S}|\right)$.

(ii) If $\mathscr{K}^{\prime}$ is a left ideal of $M_{k}(F) \bigotimes_{F} M_{k}(F)$ containing no nonzero elements of the form $A \otimes B$ and $\mathscr{S}^{\prime}$ is a basis of the vector space $\left\{X \in M_{k}(F) \mid \mathscr{K}^{\prime} X=0\right\}$, then $\mathscr{S}^{\prime}$ is a uniform insulator for $M_{k}(F)$ and $\operatorname{dim}_{F}\left(\mathscr{K}^{\prime}\right)=k^{2}\left(k^{2}-\left|\mathscr{S}^{\prime}\right|\right)$.

Proof. Let $\mathscr{S}$ and $\mathscr{K}$ be as in the lemma. Clearly $\mathscr{K}$ is a left ideal of the algebra $M_{k}(F) \otimes_{F} M_{k}(F)$. Since $\mathscr{S}$ is a uniform insulator for $M_{k}(F),(A \otimes B) \mathscr{S} \neq 0$ for all nonzero $A, B \in M_{k}(F)$ and so $\mathscr{K}$ contains no nonzero elements of the form $A \otimes B$. Write $\mathscr{S}=\left\{X_{1}, X_{2}, \ldots, X_{m}\right\}$ where $m=|\mathscr{S}|$. Define a linear map

$$
\psi_{\mathscr{S}}: M_{k}(F) \otimes_{F} M_{k}(F) \rightarrow M_{k}(F)^{m}, \quad \psi_{\mathscr{S}}(U)=\left(U X_{1}, U X_{2}, \ldots, U X_{m}\right)
$$

for all $U \in M_{k}(F) \otimes_{F} M_{k}(F)$. Clearly $\psi_{\mathscr{S}}$ is a left $M_{k}(F) \otimes_{F} M_{k}(F)$-module map and $\mathscr{K}=\operatorname{ker}\left(\psi_{\mathscr{S}}\right)$. Since $\left\{X_{1}, X_{2}, \ldots, X_{m}\right\}$ is linearly independent over $F$ and $M_{k}(F) \otimes_{F} M_{k}(F)$ is the algebra of all linear transformations of the vector space $M_{k}(F)$, we conclude that $\psi_{\mathscr{S}}$ is an epimorphism. Therefore,

$$
\begin{aligned}
\operatorname{dim}_{F}(\mathscr{K}) & =\operatorname{dim}_{F}\left(\operatorname{ker}\left(\psi_{\mathscr{S}}\right)\right)=k^{4}-\operatorname{dim}_{F}\left(\operatorname{Im}\left(\psi_{\mathscr{S}}\right)\right) \\
& =k^{4}-k^{2}|\mathscr{S}|=k^{2}\left(k^{2}-|\mathscr{S}|\right) .
\end{aligned}
$$


Further let $\mathscr{K}^{\prime}$ and $\mathscr{S}^{\prime}$ be as in the lemma. Since $\mathscr{K}^{\prime}$ is a proper left ideal of $M_{k}(F) \otimes_{F} M_{k}(F) \cong M_{k^{2}}(F)$, there exists an idempotent $E \in M_{k}(F) \otimes_{F} M_{k}(F)$ such that $\mathscr{K}^{\prime}=\left(M_{k}(F) \otimes_{F} M_{k}(F)\right) E$ and $E \neq 1$ where 1 is the identity of the algebra $M_{k}(F) \otimes_{F} M_{k}(F)$. Clearly

$$
(1-E) M_{k}(F)=\left\{X \in M_{k}(F) \mid \mathscr{K}^{\prime} X=0\right\}
$$

and so $\mathscr{S}^{\prime}$ is a basis of the vector space $(1-E) M_{k}(F)$. Write $\mathscr{S}^{\prime}=\left\{Y_{1}, \ldots, Y_{r}\right\}$ where $r=\left|\mathscr{S}^{\prime}\right|$. Consider the linear map

$$
\psi_{\mathscr{S}^{\prime}}: M_{k}(F) \otimes_{F} M_{k}(F) \rightarrow M_{k}(F)^{r}, \quad U \mapsto\left(U Y_{1}, U Y_{2}, \ldots, U Y_{r}\right) .
$$

We claim that $\operatorname{ker}\left(\psi_{\mathscr{S}^{\prime}}\right)=\left(M_{k}(F) \otimes_{F} M_{k}(F)\right) E=\mathscr{K}^{\prime}$. Indeed, the inclusion $\operatorname{ker}\left(\psi_{\mathscr{S}^{\prime}}\right) \supseteq \mathscr{K}^{\prime}$ follows from the definition of $\psi_{\mathscr{S}^{\prime}}$. Next, let $U \in \operatorname{ker}\left(\psi_{\mathcal{S}^{\prime}}\right)$. Then $U Y_{i}=0$ for all $i=1,2, \ldots, r$. Since $\left\{Y_{1}, Y_{2}, \ldots, Y_{r}\right\}$ is a basis of $(1-E) M_{k}(F)$, we conclude that $[U(1-E)] M_{k}(F)=0$. Recalling that $M_{k}(F)$ is a faithful left $M_{k}(F) \otimes_{F} M_{k}(F)$-module, we get that $U(1-E)=0$ forcing $U=U E$. That is $U \in \mathscr{K}^{\prime}$ and our claim is proved.

Since $\operatorname{ker}\left(\psi_{\mathscr{S}^{\prime}}\right)=\mathscr{K}^{\prime}$, it follows from our assumption on $K^{\prime}$ that $\operatorname{ker}\left(\psi_{\mathscr{S}^{\prime}}\right)$ contains no nonzero matrices of the form $A \otimes B, A, B \in M_{k}(F)$. That is to say, $\mathscr{S}^{\prime}$ is a uniform insulator for $M_{k}(F)$. As above we get

$$
\operatorname{dim}_{F}\left(\mathscr{K}^{\prime}\right)=\operatorname{dim}_{F}\left(\psi_{\mathscr{S}^{\prime}}\right)=k^{4}-k^{2}\left|\mathscr{S}^{\prime}\right|=k^{2}\left(k^{2}-\left|\mathscr{S}^{\prime}\right|\right)
$$

The proof is thereby complete.

PROOF OF THEOREM 1.3. Let $\mathscr{S}$ be a uniform insulator for $M_{k}(F)$ with $|\mathscr{S}|=$ $m_{k}(F)$ and let $\mathscr{V}=\sum_{A \in \mathscr{S}} F A$. According to Lemma $2.2, \mathscr{V}^{\perp}$ contains no rank one matrices and so (2) yields

$$
n_{k}(F) \geq \operatorname{dim}_{F}\left(\mathscr{V}^{\perp}\right)=k^{2}-\operatorname{dim}_{F}(\mathscr{V})=k^{2}-m_{k}(F) .
$$

That is to say $m_{k}(F)+n_{k}(F) \geq k^{2}$. On the other hand, if $\mathscr{W}$ is a subspace of $M_{k}(F)$ of dimension $n_{k}(F)$ containing no rank one matrices and $\mathscr{T}$ is a basis of $\mathscr{W}^{\perp}$, then $\mathscr{T}$ is a uniform insulator for $M_{k}(F)$ by Lemma 2.2 and so

$$
m_{k}(F) \leq|\mathscr{T}|=\operatorname{dim}_{F}\left(\mathscr{W}^{\perp}\right)=k^{2}-\operatorname{dim}_{F}(\mathscr{W})=k^{2}-n_{k}(F)
$$

forcing $m_{k}(F)+n_{k}(F) \leq k^{2}$. Therefore, $m_{k}(F)+n_{k}(F)=k^{2}$.

Let $\mathscr{K}^{\prime}$ be any left ideal of $M_{k}(F) \otimes_{F} M_{k}(F)$ containing no nonzero elements of the form $A \otimes B, A, B \in M_{k}(F)$. We claim that

$$
\operatorname{dim}_{F}\left(\mathscr{X}^{\prime}\right) \leq k^{2} \cdot n_{k}(F)
$$


Indeed, let $\mathscr{S}^{\prime}$ be a basis of the vector space $\left\{X \in M_{k}(F) \mid \mathscr{K}^{\prime} X=0\right\}$. According to Lemma $2.3, \mathscr{S}^{\prime}$ is a uniform insulator for $M_{k}(F)$ and since $\left|\mathscr{S}^{\prime}\right| \geq m_{k}(F)$,

$$
\operatorname{dim}_{F}\left(\mathscr{K}^{\prime}\right)=k^{2}\left(k^{2}-\left|\mathscr{S}^{\prime}\right|\right) \leq k^{2}\left(k^{2}-m_{k}(F)\right)=k^{2} n_{k}(F) .
$$

Now let $\mathscr{S}$ be a uniform insulator for $M_{k}(F)$ with $|\mathscr{S}|=m_{k}(F)$. It follows at once from the definition of $m_{k}(F)$ that $\mathscr{S}$ is a linearly independent subset of $M_{k}(F)$. Therefore Lemma 2.3 implies that $\mathscr{K}=\left\{U \in M_{k}(F) \otimes_{F} M_{k}(F) \mid U \mathscr{S}=0\right\}$ is a left ideal of $M_{k}(F) \otimes_{F} M_{k}(F)$ containing no nonzero elements of the form $A \otimes B$ and $\operatorname{dim}_{F}(\mathscr{K})=k^{2}\left(k^{2}-m_{k}(F)\right)=k^{2} n_{k}(F)$ by the above result. It now follows from (3) that

$$
\max \left\{\operatorname{dim}_{F}\left(\mathscr{K}^{\prime}\right)\right\}=k^{2} n_{k}(F),
$$

where $\mathscr{K}^{\prime}$ is a left ideal of $M_{k}(F) \otimes_{F} M_{k}(F)$ containing no nonzero elements of the form $A \otimes B$.

Since $M_{k}(F) \otimes_{F} M_{k}(F)$ is isomorphic to $M_{k^{2}}(F)$ under $\phi: A \otimes B \mapsto A \bullet B$ (see Section 1), we conclude from (4) that $l_{k}(F)=k^{2} \cdot n_{k}(F)$. The proof is complete.

REMARK 2.4. We conclude our discussion of the uniform bounds of primeness by considering the following implications for a field $F$ and a positive integer $k$.

(i) If $\mathscr{S}$ is a uniform insulator for $M_{k}(F)$ and $\mathscr{V}=\sum_{A \in \mathscr{S}} F A$, then $\mathscr{V}$ contains a uniform insulator $\mathscr{S}^{\prime}$ for $M_{k}(F)$ with $\left|\mathscr{S}^{\prime}\right|=m_{k}(F)$.

(ii) If $\mathscr{W}$ is a subspace of $M_{k}(F)$ maximal with respect to the property $\mathscr{W} \cap\left\{M_{k, 1}(F) \bullet M_{1, k}(F)\right\}=0$, then $\operatorname{dim}_{F}(\mathscr{W})=n_{k}(F)$.

(iii) If $\mathscr{K}$ is a left ideal of $M_{k^{2}}(F)$ maximal with respect to the property $\mathscr{K} \cap\left\{M_{k}(F) \bullet M_{k}(F)\right\}=0$, then $\operatorname{dim}_{F}(\mathscr{K})=l_{k}(F)$.

We cannot prove any of these but we show that they are equivalent:

Proof. Suppose that (i) is satisfied. We prove (ii). Let $\mathscr{W}$ be as in (ii). According to Lemma 2.2 any basis of $\mathscr{W}^{\perp}$ is a uniform insulator for $M_{k}(F)$. It now follows from our assumption that $\mathscr{W}^{\perp}$ contains a uniform insulator $\mathscr{S}^{\prime}$ for $M_{k}(F)$ with $\mathscr{S}^{\prime}=$ $m_{k}(F)$. Set $\mathscr{V}=\sum_{A \in \mathscr{S}^{\prime}} F A$ and note that $\operatorname{dim}_{F}(\mathscr{V})=m_{k}(F)$ because the set $\mathscr{S}^{\prime}$ is linearly independent. Next, the inclusion $\mathscr{V} \subseteq \mathscr{W}^{\perp}$ together with (2) yield that $\mathscr{V}^{\perp} \supseteq\left(\mathscr{W}^{\perp}\right)^{\perp}=\mathscr{W}$. By Lemma $2.2 \mathscr{V}^{\perp}$ contains no rank 1 matrices and so the maximality of $\mathscr{W}$ implies that $\mathscr{V}^{\perp}=\mathscr{W}$. Therefore $\mathscr{V}=\left(\mathscr{V}^{\perp}\right)^{\perp}=\mathscr{W}^{\perp}$ and so $\operatorname{dim}_{F}\left(\mathscr{W}^{\perp}\right)=\operatorname{dim}_{F}(\mathscr{V})=m_{k}(F)$. Recalling that $\operatorname{dim}_{F}(\mathscr{W})=k^{2}-\operatorname{dim}_{F}\left(\mathscr{W}^{\perp}\right)=$ $k^{2}-m_{k}(F)$, we conclude that $\operatorname{dim}_{F}(\mathscr{W})=n_{k}(F)$ by Theorem 1.3.

Now assume that (ii) is fulfilled and show that (i) is true. Let $\mathscr{S}$ and $\mathscr{V}$ be as in (i). Then $\mathscr{V}^{\perp}$ contains no rank 1 matrices by Lemma 2.2. Let $\mathscr{W}$ be a subspace of $M_{k}(F)$ 
containing $\mathscr{V}^{\perp}$ and maximal with respect to the property $\mathscr{W} \cap\left\{M_{1 k}(F) \bullet M_{1 k}(F)\right\}=0$. By our assumption $\operatorname{dim}_{F}(\mathscr{W})=n_{k}(F)$ and so (2) together with Theorem 1.3 imply that $\mathscr{V}=\left(\mathscr{V}^{\perp}\right)^{\perp} \supseteq \mathscr{W}^{\perp}$ and $\operatorname{dim}_{F}\left(\mathscr{W}^{\perp}\right)=k^{2}-n_{k}(F)=m_{k}(F)$. Let $\mathscr{S}^{\prime}$ be a basis of $\mathscr{W}^{\perp}$. Then $\mathscr{S}^{\prime}$ is a uniform insulator for $M_{k}(F)$ by Lemma 2.2. Clearly $\left|\mathscr{S}^{\prime}\right|=m_{k}(F)$ and $\mathscr{S}^{\prime} \subseteq \mathscr{V}$.

Finally, making use of Lemma 2.3 the proof of the equivalence of statements (i) and (iii) is similar to that of (i) and (ii).

\section{Acknowledgements}

The authors express their gratitude to John van den Berg, University of Natal, South Africa, for attracting their attention to the subject and stimulating discussions and to the referee of the paper for his valuable remarks.

\section{References}

[1] D. Handelman and L. Lawrence, 'Strongly prime rings', Trans. Amer. Math. Soc. 211 (1975), 209-223.

[2] J. E. van den Berg, 'On uniformly strongly prime rings', Math. Japon. 38 (1993), 1157-1166.

[3] —_, 'A note on uniform bounds of primeness in matrix rings', J. Austral. Math. Soc. Ser. A 65 (1998), 212-223.

Department of Mathematics

National Cheng Kung University

Tainan

Taiwan

e-mail: beidar@mail.ncku.edu.tw
Mathematical Institute University of Düsseldorf Germany e-mail:wisbauer@math.uni-duesseldorf.de 